\title{
Phenotype and Function of Macrophage Polarization in Monocrotaline-Induced Pulmonary Arterial Hypertension Rat Model
}

\author{
Yong FAN ${ }^{1}$, Yanjie HAO ${ }^{1}$, Dai GAO ${ }^{1}$, Lan GAO ${ }^{2}$, Guangtao LI $^{1}$, Zhuoli ZHANG ${ }^{1}$ \\ ${ }^{1}$ Department of Rheumatology and Clinical Immunology, Peking University First Hospital, Beijing, \\ China, ${ }^{2}$ Department of Rheumatology, Beijing Shijitan Hospital, Capital Medical University, \\ Beijing, China
}

Received February 28, 2020

Accepted January 26, 2021

Epub Ahead of Print March 8, 2021

\section{Summary}

Pulmonary arterial hypertension (PAH) is a fatal disease characterized by vascular remodeling and chronic inflammation. Macrophages are the key orchestrators of inflammatory and repair responses, and have been demonstrated to be vital in the pathogenesis of PAH. However, specific phenotype of macrophage polarization ( $M 1$ \& M2 macrophage) in the development of $\mathrm{PAH}$ and the underlying mechanisms how they work are still largely unclear. A rat model of monocrotaline (MCT) induced PAH was used. Hemodynamic analysis and histopathological experiments were conducted at day 3, 7, 14, 21 and 28 , respectively. In PAH rat lung tissue, confocal microscopic images showed that CD68+NOS2+ M1-like macrophages were remarkably infiltrated on early stage, but dramatically decreased in mid-late stage. Meanwhile, CD68+CD206+ M2-like macrophages in lung tissue accumulated gradually since day 7 to day 28 , and the relative ratio of M2/M1 macrophage increased over time. Results detected by western blot and immunohistochemistry were consistent. Further vitro functional studies revealed the possible mechanism involved in this pathophysiological process. By using Transwell co-culture system, it was found that M1 macrophages induced endothelial cell apoptosis, while M2 macrophages significantly promoted proliferation of both endothelial cell and smooth muscle cell. These data preliminarily demonstrated a temporal dynamic change of macrophage M1/M2 polarization status in the development of experimental PAH. M1 macrophages participated in the initial stage of inflammation by accelerating apoptosis of endothelial cell, while M2 macrophages predominated in the reparative stage of inflammation and the followed stage of aberrant tissue remodeling.

\section{Key words}

Pulmonary arterial hypertension - Macrophage polarization • Dynamic change $\bullet$ Endothelial cell $\bullet$ Smooth muscle cell

\section{Corresponding author}

Zhuoli Zhang, Department of Rheumatology and Clinical Immunology, Peking University First Hospital, No.8, Xishiku Street, West District, Beijing 100034, China. Email: zhuoli.zhang@126.com

\section{Introduction}

Pulmonary arterial hypertension (PAH) is a progressive, devastating cardiopulmonary disease characterized by increased mean pulmonary arterial pressure, pulmonary vascular remodeling and right ventricular failure (Thenappan et al. 2018). Currently, advances in therapies significantly improve clinical outcomes of PAH patients, but have little impact on the progression of pulmonary vascular remodeling. Although increased cell proliferation (e.g. endothelial cell, smooth muscle cell) has been identified in pulmonary vascular remodeling, a paradoxical increase in endothelial apoptosis is important for the initiation and progression of PAH disease (Sakao et al. 2006, Farkas et al. 2019). However, mechanisms causing endothelial cell apoptosis are only partially understood. During last two decades, there is a growing appreciation that pulmonary vascular inflammation plays a central role in the development of PAH vascular remodeling (Rabinovitch et al. 2014). An expanding body of knowledge has related 
macrophage, which is the major cell type contributing to local lung inflammation and tissue repair, to $\mathrm{PAH}$ pathogenesis. Emerging data indicated that lung macrophages were elevated prominently in experimental and clinical PAH (Savai et al. 2012, Frid et al. 2006), and macrophage inactivation prevented $\mathrm{PAH}$ in several animal models (Tian et al. 2013, Thenappan et al. 2011). More importantly, a recent study found that depletion of alveolar macrophages by clodronate-containing liposomes attenuated hypoxia-induced pulmonary hypertension (PH) (Zaloudikova et al. 2016).

It is well-established that macrophages can be classified into classically activated macrophage (M1) and alternatively activated macrophage (M2). M1 macrophage is characterized by high expression of iNOS (also NOS2) and proinflammatory factors including IL-1 $\beta$, IL-12, which leads to protective immunity against pathogens as well as immune-mediated tissue damage in uncontrolled situations. In contrast, M2 macrophage expresses high levels of CD206 (also known as mannose receptor 1) and the anti-inflammatory cytokine IL-10. Usually, M2 macrophage exhibits physiologically protective properties due to phagocytosis of debris, promoting tissue repair and termination of inflammation by downregulating M1 responses. However, under pathological condition, M2 macrophages play essential roles in the promotion of aberrant tissue remodeling, vasculogenesis and tumor progression (Murray et al. 2014).

Macrophages maintain their plasticity and alter their phenotype depending on different environmental conditions. For example, M1 macrophage can repolarize toward M2 after the phagocytosis of apoptotic cell (Freire-de-Lima et al. 2006), suggesting that reprogramming inflammatory M1 macrophages toward an M2 phenotype may be involved in the resolution phase of some certain disease, like atherosclerosis (Tabas and Bornfeldt 2016), infectious diseases (Davis et al. 2013), multiple sclerosis (Herder et al. 2015) and psychiatric disorders (Nakagawa and Chiba 2014). However, whether there is a temporal change of macrophage M1/M2 polarization in the pathogenesis of PAH is still unclear. Furthermore, the mechanism on how these two different macrophage subtypes participated in the pathogenesis of PAH vascular remodeling have not yet been elucidated.

In the present study, we aimed to explore these questions by dynamically analyzing the changes of macrophage polarization phenotype (M1/M2) in lung tissue in monocrotaline-induced PAH (MCT-PAH) rat model, and preliminarily investigating the underlying biological function of different activated macrophages on pulmonary arterial smooth muscle cells and endothelial cells in vitro.

\section{Materials and Methods}

\section{Animal experiments}

Male Sprague-Dawley rats $(180-200 \mathrm{~g})$ were used for the monocrotaline-induced PAH model as previously described (Gao et al. 2017). Briefly, the animals were randomly divided into two groups: MCT-PAH group and Control group by a single subcutaneous injection of MCT or saline. After the injection, rats from these two groups were sacrificed at five time points: Day 3, 7, 14, 21 and day 28 postinjection ( $\mathrm{n}=8$ for each subgroup). All procedures were performed according to the protocols approved by Animal Research Ethics Committee of Peking University Health Science Center (permit number J201527) and the Guide for the Care and Use of Laboratory Animals published by the US National Institutes of Health (NIH Publication Number 85-23, revised 1996).

\section{Right heart catheterization (RHC)}

Pulmonary artery pressure was measured by RHC method described previously (Gao et al. 2017). Briefly, the animals were initially anesthetized by using pentobarbital sodium via intraperitoneal injection (60 mg $\cdot \mathrm{kg}-1$, Sigma, USA) and a tracheotomy performed. A right heart catheter (PE-50 tubing) was inserted through the right jugular vein to measure the right ventricular systolic pressure (RVSP) and mean pulmonary arterial pressure (mPAP).

\section{Tissue preparation}

After RHC, the chest was opened and the right hilus pulmonis was ligated. The right lung of each animal was excised and divided by the upper and lower lobes. Each upper lobe was fixed using $10 \%$ formaldehyde, embedded in paraffin or frozen Optimal Cutting Temperature (OCT) compound, and then sectioned $(5 \mu \mathrm{m}$ thickness). Sections were stained for hematoxylin and eosin (H\&E) staining, as well as immunohistochemistry/immunofluorescence staining. Each lower lobe was frozen in liquid nitrogen for Western blot analysis. After that, the heart of each rat was isolated. The right ventricular (RV) free wall was dissected from the left 
ventricle plus interventricular septum $(\mathrm{LV}+\mathrm{S})$ and weighed separately. The degree of right ventricular hypertrophy $(\mathrm{RVH})$ was determined by the weight ratio: $\mathrm{RV} /(\mathrm{LV}+\mathrm{S})$.

\section{Histological analysis and morphometric analysis}

Standard histopathological procedures were used to prepare 5- $\mu \mathrm{m}$-thick sections. The sections were deparaffinized and stained with hematoxylin and eosin (ZSGB-BIO, Beijing, China). The lungs were examined with light microscopy for morphological alterations by two separate examiners. Morphometric analyses were performed in pulmonary arteries with an external diameter $<100 \mathrm{~mm}$. The pulmonary arterial medial wall thickness was calculated as \% wall thickness (WT \%) = (wall thickness $\times 2 /$ external diameter) $\times 100$. For quantitative analyses, the average pulmonary arterial medial wall thickness of 15 vessels from each rat was calculated. The diameter of pulmonary arteries was determined by Image $\mathbf{J}$ software (National Institutes of Health).

Immunohistochemical and immunofluorescence staining For immunohistochemical staining (IHC), $5 \mu \mathrm{m}$ lung sections were deparaffinized, hydrated and incubated in $10 \mathrm{mM}$ sodium citrate buffer at a microwave oven (a total of 10 minutes) for antigen retrieval. Sections were incubated overnight at $4{ }^{\circ} \mathrm{C}$ with a primary antibody against CD68 (sc-59103, Santa Cruz Biotechnology), IL-10 (ab9969, Abcam) and IL-12p40 (NBP1-20706, Novus Biologics). After extensive washing in PBS buffer, sections were incubated with HRP-coupled secondary antibodies for $60 \mathrm{~min}$, and were visualized using 3,3- diaminobenzidine and counter stained with hematoxylin (ZSBG-BIO, Beijing, China). Control experiments included omission of the primary antibodies and substitution of the primary antibodies with nonimmune rabbit or mouse IgG. Immunohistochemical staining was examined by an Olympus BX51 microscope (Olympus, Japan) and analyzed by Image Pro-Plus software.

For immunofluorescence staining (IF), lungs were fixed with $4 \%$ paraformaldehyde overnight at $4{ }^{\circ} \mathrm{C}$ and embedded in OCT. Briefly, NOS2 (ab15323, 1:200, Abcam) and CD206 (ab64693, 1:500, Abcam) markers were used to identify M1 and M2 macrophages in lung tissue, respectively. CD68 (sc-59103, 1:100, Santa Cruz Biotechnology) was used as a pan-macrophage marker. Serial sections were prepared and incubated with indicated primary antibodies overnight at $4{ }^{\circ} \mathrm{C}$ and then with a 1:200 dilution of Alexa Fluor 488 or 594-conjugated IgG (Invitrogen) for $1 \mathrm{~h}$ at room temperature. Sections were counterstained with Mounting Medium with 4', 6-diamidino-2-phenylindole (DAPI, ZSGB-BIO, Beijing, China) and cover slipped. The images were captured with a TCS SP5 confocal microscope (Leica, Germany) in the Center of Medical and Health Analysis faculty of Peking University Health Science Center.

\section{Western blot analyses}

Lung tissues or cells were lysed using radioimmunoprecipitation assay buffer (RIPA) with $100 \mu \mathrm{g} / \mathrm{ml}$ phenylmethylsul fonylfluoride (PMSF). Then, 20 $\mu \mathrm{g}$ of protein from cell lysates was electrophoresed on 6-12\% SDS-polyacrylamide gels and transferred to an immobilon PVDF membrane (Millipore, Billerica, MA, USA). Nonspecific antibody binding was blocked using $5 \%$ skim milk in TBS-T, and the membranes were incubated with primary anti-NOS2, anti-CD206, anti-IL6, anti-IL10 and anti- $\beta$-actin antibodies overnight at $4{ }^{\circ} \mathrm{C}$. Anti-mouse or anti-rabbit (1: 5000) horseradish peroxidase-conjugated secondary antibodies were incubated for $1 \mathrm{~h}$ at room temperature. Antibodies were visualized using an ECL solution kit (Millipore, Billerica, MA, USA) in an imager (Bio-Rad Laboratories, Hercules, CA, USA). Quantification of relative changes in protein levels (relative band intensity, expressed as percentage of control protein levels) were performed using the Image $\mathrm{J}$ software.

\section{Quantitative real-time polymerase chain reaction (qRT-PCR)}

Total RNA from lung tissues or cells was isolated using Trizol Reagent according to the manufacturer's instructions. cDNA was synthesized using a Reverse Transcriptase kit (Invitrogen, USA). qRT-PCR was performed using SYBR Green PCR Master Mix with the ABI-prism 7500 system (Applied Biosystems, USA) in triplicate. The relative gene expression level was normalized for expression of the $\beta$-actin and calculated using the comparative $\mathrm{Ct}$ method formula 2- $\Delta \Delta \mathrm{CT}$ method (Livak and Schmittgen 2001). The primer sequences are shown in Table 1.

\section{Cell culture and macrophage differentiation}

Pulmonary arterial smooth muscle cells (PASMCs) were obtained from wild-type rat intra- 
pulmonary arteries (the 3rd or 4th division) as previously described (Gao et al. 2017). Briefly, left rat lungs were removed and transferred to a dish full of cold physiological salt solution. Intrapulmonary arteries were gently isolated, and then arteries adventitia and endothelium were carefully cleaned under a microscope. The resting media tissue were digested by type I collagenase and papain. Human umbilical vein endothelial cells (HUVECs) were purchased from the cell bank of the Chinese Academy of Sciences (Beijing, People's Republic of China, http://english.cas.cn) and RAW264.7 macrophage cell line was kindly provided by Professor Yuedan Wang (Peking University Health Science Center). PASMCs were identified by $\alpha$-smooth muscle actin (SMA) via immunofluorescence staining while HUVECs were identified by von Willebrand factor (vWF). All these cells were cultured in the DMEM medium supplemented with $10 \%$ fetal bovine serum (FBS), penicillin (100 units/ml), and streptomycin (100 units $/ \mathrm{ml}$ ) in a humidified atmosphere with $5 \% \mathrm{CO}_{2}$ at $37^{\circ} \mathrm{C}$. Culture mediums were changed every 2 to 3 days on average. The M1 and M2 macrophages were prepared by stimulating RAW264.7 macrophages with LPS (100ng/ml, PeproTech)+IFN- $\gamma(20 \mathrm{ng} / \mathrm{ml}$, PeproTech) or IL-4 (20ng/ml, PeproTech)+IL-13 (20ng/ml, PeproTech) for 24 hours, respectively. Untreated RAW264.7 cells were used as control M0 macrophage. The phenotype of the subsets were identified through Western blot and qRT-PCR analysis for gene signatures of representative markers.

\section{Cell viability assay}

The cell viability was evaluated by Cell Counting Kit-8 (CCK8) assay (Dojindo Molecular Technologies, Inc.). Briefly, PASMCs (or HUVECs) were seeded at $5 \times 10^{\wedge} 3$ cells per well in 96 -well plates overnight, after placing in serum-starved condition for a further 24 hours, various concentration of supernatant from different types of macrophages were added and incubated for 72 hours (supernatants were diluted 1:0, 1:1 and 1:3 with normal culture medium and daily refreshed). Cell stimulated with normal culture medium was set as control. 10ul of CCK8 solution was added into the well and incubated for an additional 2 hours, then the optical density (OD) for each well was measured at a wavelength of $450 \mathrm{~nm}$. The cell viability (\% of control) is expressed as the percentage of (ODtest - ODblank) / (ODcontrol ODblank).

Transwell co-cultures system and analyses of cell cycle and apoptosis

The different types of macrophages and PASMCs (or HUVECs) were placed in Transwell coculture chambers separated by membrane filter that prevented direct cell-to-cell contact. Briefly, the transwell insert was added to the 6-well plates with PASMCs (or HUVECs, $2 \times 10^{\wedge} 5$ per well) in the bottom, and then $5 \times 10^{\wedge} 4$ macrophages were added in the upper, meanwhile untreated PASMCs (or HUVECs) served as the control group. After $72 \mathrm{~h}$ of incubation, the PASMCs (or HUVECs) were collected and used for the subsequent research. For cell cycle analysis, PASMC (or HUVEC) were harvested, washed twice with PBS and fixed with $70 \%$ cold ethanol for at least 3 hours. Then the fixed cells were washed once with PBS and stained using Propidium Iodide for 30 minutes in the dark and analyzed by flow cytometry. Similarly, cell apoptosis analysis was detected by staining live cells with FITC-conjugated Annexin- $\mathrm{V}$ and Propidium Iodide (Keygen, Biotech, Beijing, China) and analyzed by flow cytometry as well. All analyses were carried out in three replicates.

Table 1. Lists of qRT-PCR primers (mouse)

\begin{tabular}{lcc}
\hline Gene & \multicolumn{1}{c}{ Forward primer } & Reverse primer \\
\hline Beta-actin & 5'-AGGCCAACCGTGAAAAGATG-3' & 5'-CCAGAGGCATACAGGGACAAC-3' \\
TNF- $\alpha$ & 5'-CAGGCGGTGCCTATGTCTC-3' & 5'-CGATCACCCCGAAGTTCAGTAG-3' \\
IL-6 & 5'-CTGCAAGAGACTTCCATCCAG-3' & 5'-AGTGGTATAGACAGGTCTGTTGG-3' \\
NOS2 & 5'-GTTCTCAGCCCAACAATACAAGA-3' & 5'-GTGGACGGGTCGATGTCAC-3' \\
CD206 & 5'-GGCTGATTACGAGCAGTGGA-3' & 5'-CATCACTCCAGGTGAACCCC-3' \\
IL-10 & 5'-GCTGCCTGCTCTTACTGACT-3' & 5'-CTGGGAAGTGGGTGCAGTTA-3' \\
Arginase-1 & 5'-AGCACTGAGGAAAGCTGGTC-3' & 5'-CAGACCGTGGGTTCTTCACA-3' \\
Fizz1 & 5'-GACTGCTACTGGGTGTGCTT-3' & 5'-GCTGGGTTCTCCACCTCTTC-3' \\
\hline
\end{tabular}




\section{Statistical analysis}

Data were expressed as means \pm standard deviation and analyzed using independent $t$ tests (for parametric data) or Mann-Whitney $U$ tests (for nonparametric data). Statistical comparison of multiple groups was performed by one-way ANOVA with Bonferroni post-hoc test or Kruskal-Wallis test followed by Dunn's multiple comparisons. A two-tailed $\mathrm{p}$ value less than 0.05 was considered to be statistically significant.
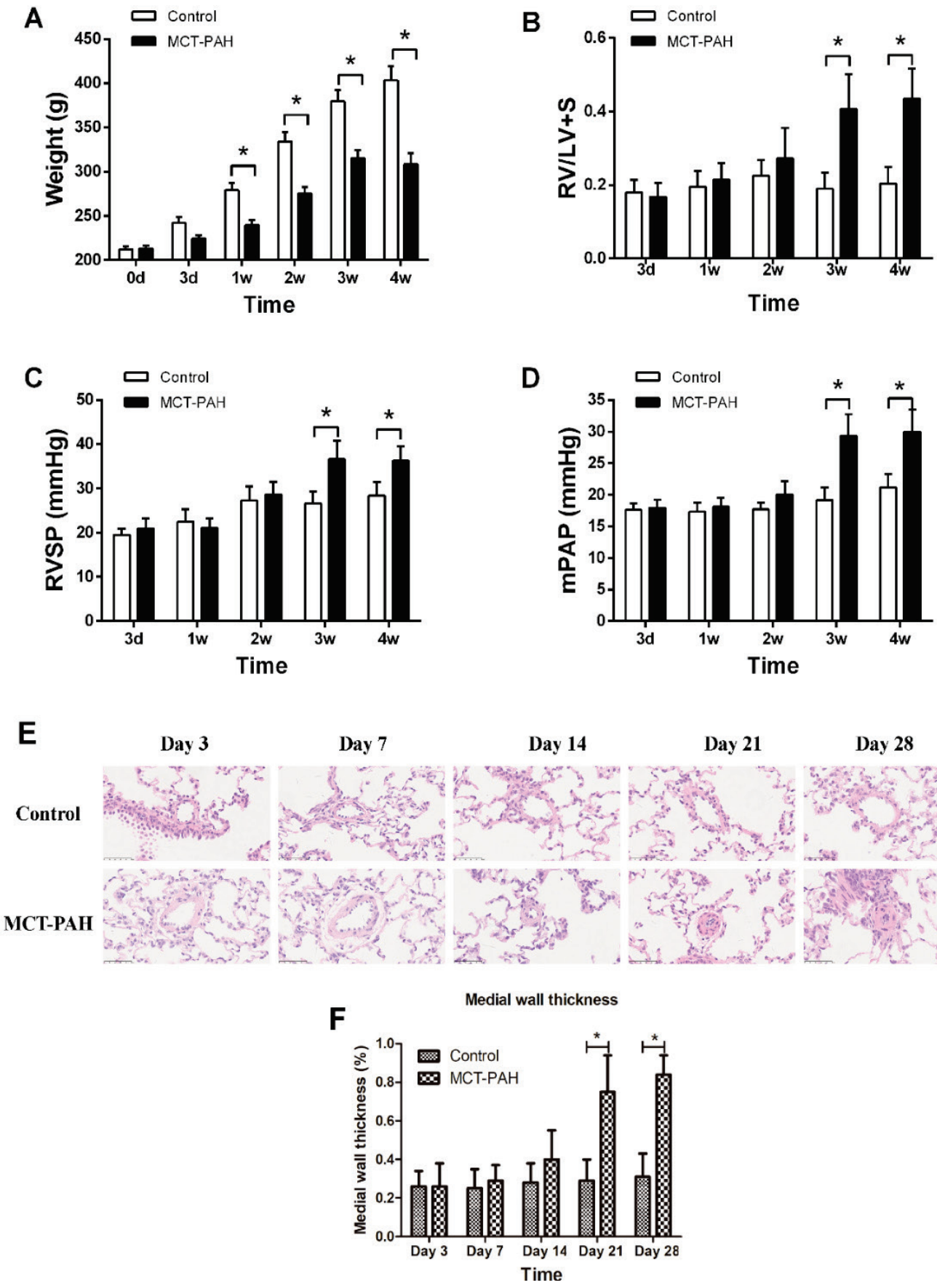

Fig. 1. Temporal dynamic change of monocrotaline-induced PAH rat model. (A) Temporal dynamic change of rat weight in the development of MCT-PAH. It was found that MCT treatment resulted in significantly decreasing body weight just after 1 week. (B) Temporal dynamic change of right ventricular hypertrophy index (RVHI: RV/LV+S) in the development of MCT-PAH. Evident right ventricular hypertrophy occurred 3 weeks after MCT injection. (C) Temporal dynamic change of right ventricular systolic pressure (RVSP) in the development of MCT-PAH. (D) Temporal dynamic change of mean pulmonary arterial pressure (mPAP) in the development of MCT-PAH. Both RVSP and MPAP were markedly elevated after 3 weeks in MCT treated rats. (E) Representative images of H\&E-stained lung sections with vessel walls shown at 400 -fold magnification. Scale bars $=50 \mu \mathrm{m}$. (F) Quantitative analysis of pulmonary arterial medial wall thickness. There was no apparent pulmonary vascular remodelling until day 21 (3 weeks) post-MCT. 15 vessels from each rat were counted and the average was calculated. $\mathrm{N}=8$ rats per subgroup. Data represent mean \pm standard deviation. $* \mathrm{P}<0.05$ vs. Control (tested by independent $\mathrm{t}$ tests). 
Normal control MCT 7d MCT 14d

(A)
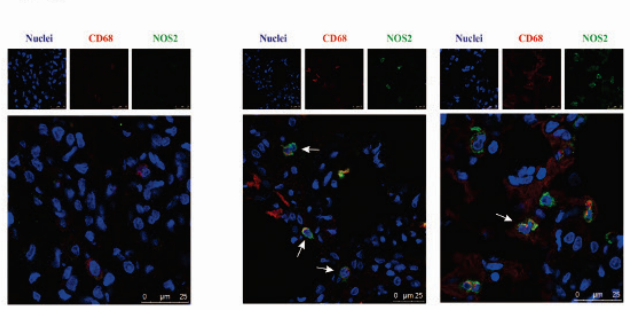

MCT 21d
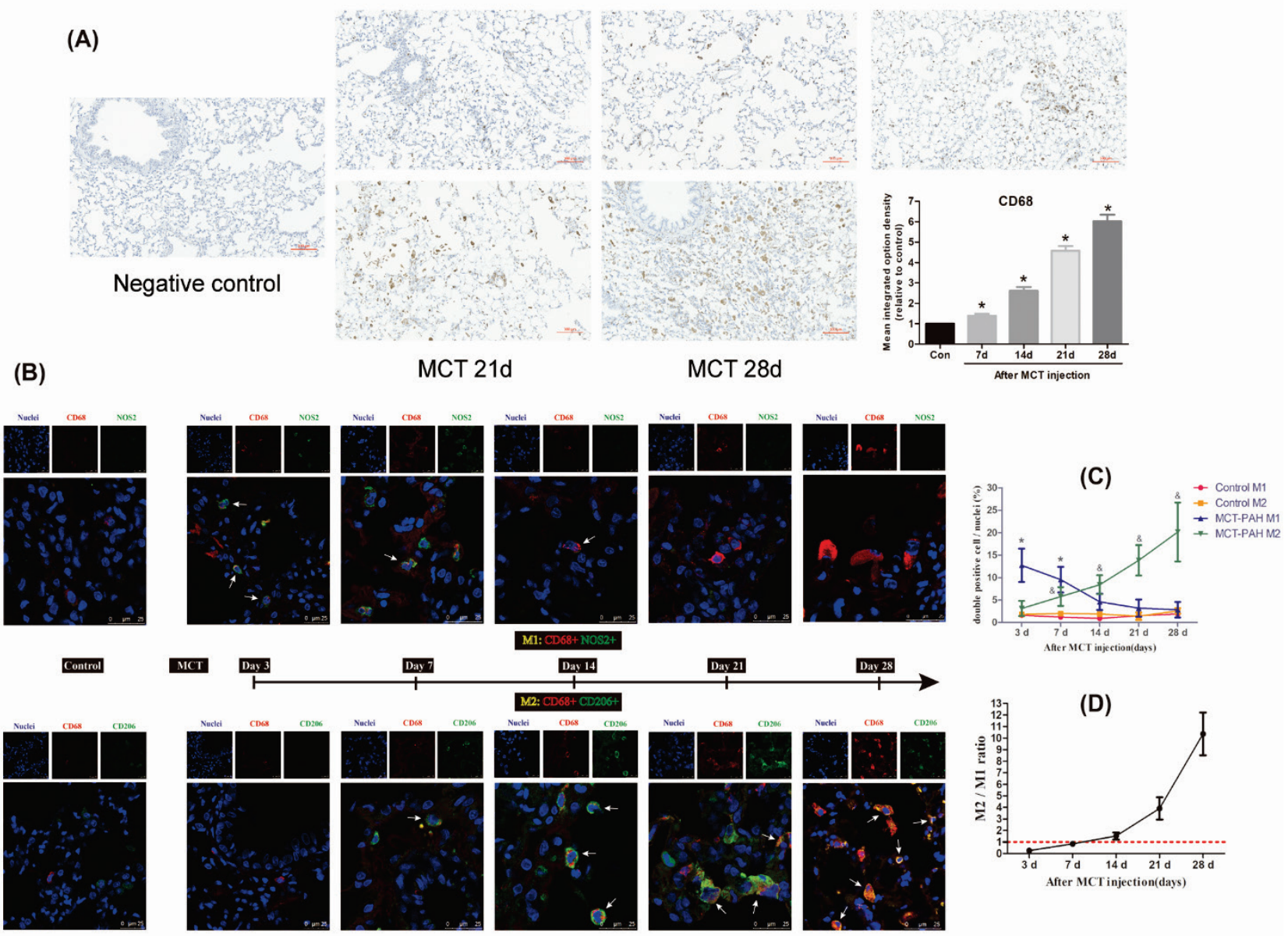

Fig. 2. Pulmonary macrophage in lung tissue. (A) Representative images of immunohistochemistry staining showed that CD68+ macrophage were mainly observed in perivascular space and alveolar space, and these cells accumulated over time from day 7 to day 28 (4 weeks) after exposed to MCT. Scale bars $=100 \mu \mathrm{m}$. (B) Representative confocal images of triple staining assessed by immunofluorescence staining (DAPI for nuclei, CD68 for pan-macrophage marker, NOS2 for M1 macrophage marker and CD206 for M2 macrophage marker). White arrow represents double positive macrophage. Scale bars $=25 \mu \mathrm{m}$. (C) Semiquantitative proportion of double-stained positive cells in total cells. The M1 proportion in MCT-PAH group increased at the early stage and decreased at the mid-late stage, whereas the M2 proportion increased over time from early to late stage. ${ }^{*} \mathrm{P}<0.05$ vs. Control M1 proportion (tested by independent $\mathrm{t}$ tests); ${ }^{\&} \mathrm{P}<0.05$ vs. Control M2 proportion (tested by independent $\mathrm{t}$ tests). (D) Relative ratio of the M2/M1 macrophage during the development of $\mathrm{PAH}$. The ratio of M2/M1 increased over time and was much more pronounced at the late stage.

\section{Results}

Temporal dynamic change of MCT-induced PAH rat model

To assess dynamic change of hemodynamic parameters, vascular remodeling and right ventricle hypertrophy during the development of MCT-induced PAH rat model, RHC and HE staining were conducted at day 3, 7, 14, 21, 28 after MCT or saline injection. MCT treatment resulted in significantly decreasing body weight just after 1 week of injection, and suddenly jumping up of RVSP and mPAP compared with the control rats after 2 weeks of injection (Fig. 1A/C/D). Accompanied with increased pressures, right ventricle reconstructed and became hypertrophic as indicated by the progressive increase of $\mathrm{RV} /(\mathrm{LV}+\mathrm{S})$ weight ratio after 2 weeks (Fig. 1B). Fig. 1E/F displayed the representative HE-stained lung sections to show the histological changes. In monocrotaline induced animals, the percentage of pulmonary arterial medial wall thickness (diameter $<100 \mathrm{~mm}$ ) was first significantly increase at day 21 , and further slightly grow up at day 28 . There was no evidence of vascular remodeling at 1 or 2 weeks postMCT, which was generally consistent with the hemodynamic data. 
(A)

(A)

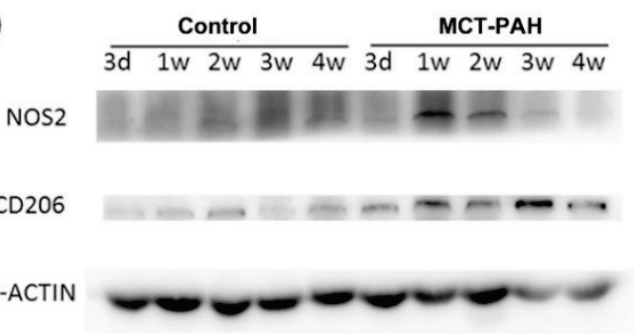

(B)

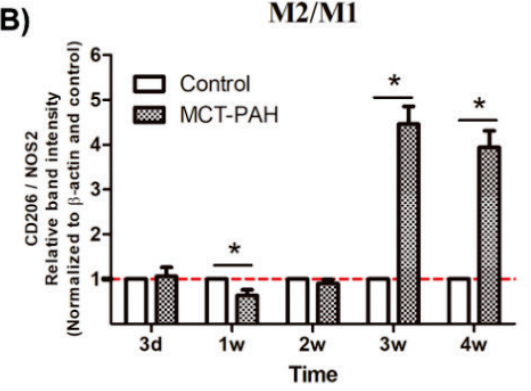

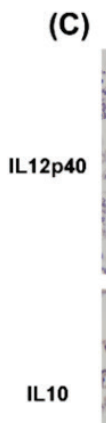

(C)

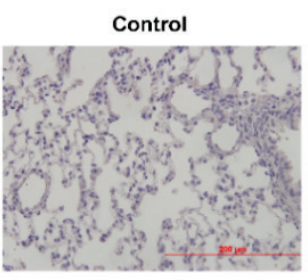

IL10

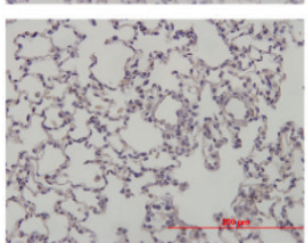

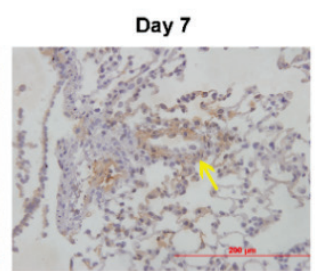

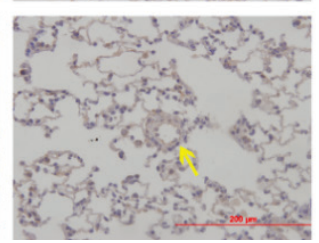

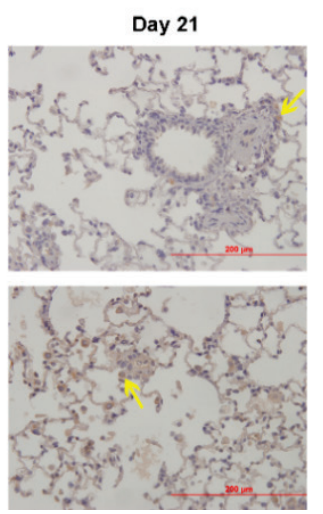

Fig. 3. Temporal dynamic expression of M1- and M2- polarization markers in lung tissue. (A-B) Representative western blotting and quantitative analysis showing the protein levels of NOS2 (M1 marker) and CD206 (M2 marker). The relative band intensity ratio of CD206/NOS2 was less than 1 at day 7 post-MCT, however, markedly elevated at third and fourth week. (C-D) Representative immunohistochemical images and quantitative analysis showing the expression of IL12 (M1 marker) and IL10 (M2 marker). M1-associated proinflammatory cytokine IL12 was highly expressed at the early stage of PAH while M2-associated anti-inflammatory cytokine IL-10 was detected mainly at the late stage. Yellow arrows indicate IL-12 or IL-10 positive staining of cells. Scale bars = $200 \mu \mathrm{m} . \mathrm{N}=8$ rats per subgroup. Data represent mean \pm standard deviation. $* \mathrm{P}<0.05$ vs. Control group or indicated object (tested by independent $\mathrm{t}$ tests).

Recruitment of CD68+ macrophages and temporal dynamic change of M1/M2 macrophages in lung tissue

To examine the distribution and intensity of macrophages in PAH lung tissue, we first did IHC staining on paraffin sections. It was showed that CD68 positive macrophages in $\mathrm{PAH}$ lung tissue were mainly observed in perivascular space and alveolar space, and these cells accumulated gradually from day 7 to the 4 th week after exposed to MCT (Fig. 2A). The mean optical density of CD68 positive cells in lung tissue increased by fivefold at 4 weeks post-MCT compared with normal control.

Then we tried to explore dynamic macrophage polarization phenotype during the development of $\mathrm{PAH}$ and dual-immunofluorescence staining for M1 and M2 macrophages were performed. Confocal microscopic images showed that CD68+NOS2+ M1-like macrophage were remarkably infiltrated since day 3 after MCT injection, remained high until day 7 , and dramatically decreased from day 14. Meanwhile, CD68+CD206+ M2-like macrophage in PAH rat lung tissue accumulated since day 7 post-MCT and increased over time to day 28 (Fig. 2B). The ratio of M2/M1 macrophage was much more pronounced at the late stage than the early stage (Fig. 2C/D).

To further investigate dynamic protein expression of M1- and M2- polarization signatures in lung tissue, Western blot analysis and IHC staining were conducted. As shown in Fig. 3A/B, the M1 marker (NOS2) was highly expressed on day 7 after MCT injection and remained detectable on day 14, while M2 marker (CD206) expressed slightly higher from day 7 and peaked on day 21 . The ratio of M2/M1 was less than 1 at day 7 post-MCT, however, progressively elevated after 3 weeks. Immunohistochemistry showed that the M1-associated proinflammatory cytokine IL-12 was highly expressed at the early stage (day 7 after MCT injection) of PAH compared to that of the late stage (day 21 after MCT injection). Conversely, the expression of the M2-associated anti-inflammatory cytokine IL-10 was detected mainly at the late stage rather than early stage (Fig. 3C/D). Given the above, these data indicated an alteration of M1 and M2 macrophage in the development of PAH and a potential association between M2/M1 ratio with disease severity and vascular remodeling. 
(A)

A)

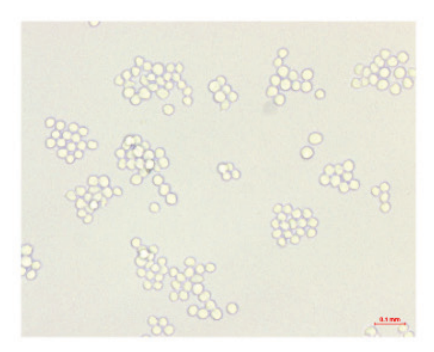

M1

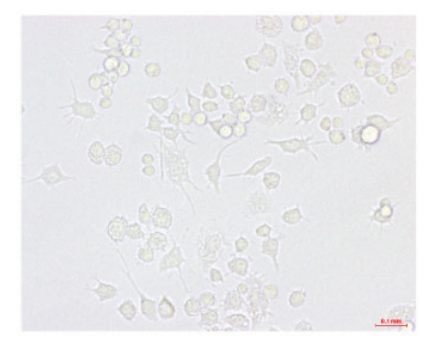

M2

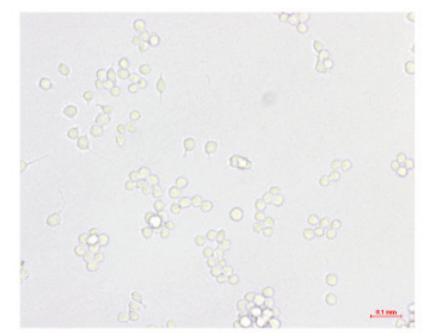

(B)
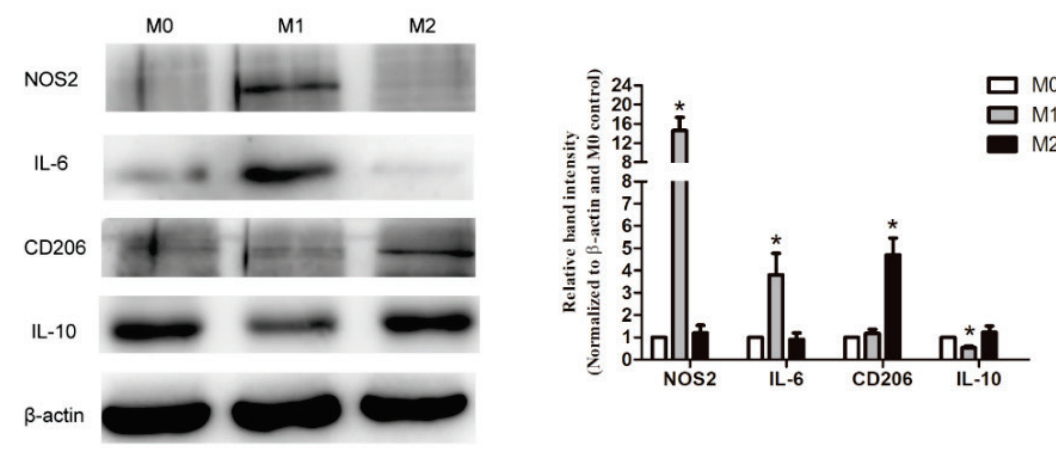

(C)

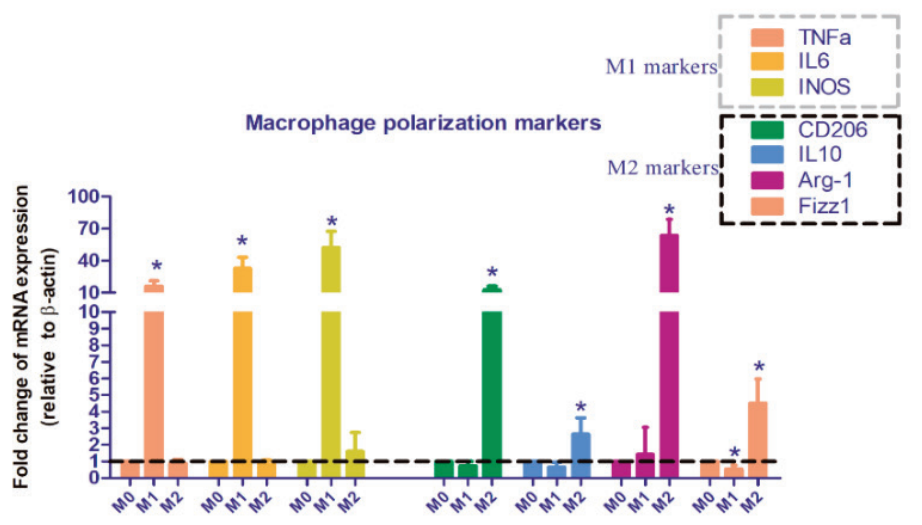

Fig. 4. Differentiation of $M 1$ and $M 2$ macrophages in vitro. (A) Cell morphology of different macrophages. M0 macrophage had a circular morphology while M1 macrophages were predominantly spindle-like and polygon shaped, and M2 macrophages showed a mixture of round and spindle shaped cells. Scale bars $=100 \mu \mathrm{m}$. (B) Western Blot analysis of M1 markers (NOS2, IL6) and M2 markers (CD206, IL10). NOS2 and IL-6 expression were enhanced in M1 macrophage while IL-10 decreased. M2 macrophage showed a higher expression of CD206. (C) qRT-PCR analysis of M1 markers (TNFa, IL-6 and NOS2) and M2 markers (CD206, IL-10, Arg-1 and Fizz-1). The expression of M1 polarization markers (TNFa, IL-6 and NOS2) were significantly increased in M1 macrophages while M2 polarization markers (CD206, IL-10, Arg-1 and Fizz-1) were highly up-regulated in M2 macrophages. Data represent mean \pm standard deviation. *P $<0.05$ vs. M0 macrophage (tested by independent $\mathrm{t}$ tests).

\section{Differentiation of M1 and M2 macrophages in vitro}

By now we still have little knowledge about the potential function of different activated macrophages on vascular remodeling in PAH. In order to shed light on this field, we tried to have some vitro functional studies. Firstly, M1 and M2 macrophages were induced in vitro by using above-mentioned protocol. The M0, M1 and M2 had different morphological characteristics. While M0 macrophage showed a circular morphology, M1 macrophages activated by LPS plus IFN- $\gamma$ were predominantly spindle-like and polygon shaped, and M2 macrophages activated by IL4 plus IL13 had a mixture of round and spindle shape (Fig. 4A). Then the Western Blot analysis showed NOS2 and IL-6 expression were enhanced in M1 macrophage while IL-10 decreased. M2 macrophage showed a higher expression of CD206 (Fig. 4B). Of the genes related to polarized macrophage, TNF $\alpha$, IL-6 and NOS2 expression were significantly increased in M1 macrophage than M0 macrophage, and CD206, IL-10, Arg-1 and Fizz-1 were up-regulated in IL4/IL13 stimulated M2 macrophage (Fig. 4C). These results together indicated that stimulation of RAW264. 7 cells with LPS+IFN- $\gamma$ or IL-4+IL-13 is a successful method to acquire M1 and M2 macrophage in vitro. 
Effects of activated macrophage on vascular smooth muscle cell and endothelial cell

After differentiation of macrophage, we then aim to evaluate the effect of activated macrophages on the cell viability of PASMCs and HUVECs. Cell identification showed a high purity of PASMCs and HUVECs (Fig. 5A). After 72 hours of treatment, supernatants from
M2 macrophages would promote cell proliferation of both PASMCs and HUVECs, which was detected by the CCK-8 assay. However, supernatants from M1 macrophages exerted a concentration-dependent decrease in the cell viability of HUVECs while had no significant effect on PASMC (Fig. 5B).
(A)

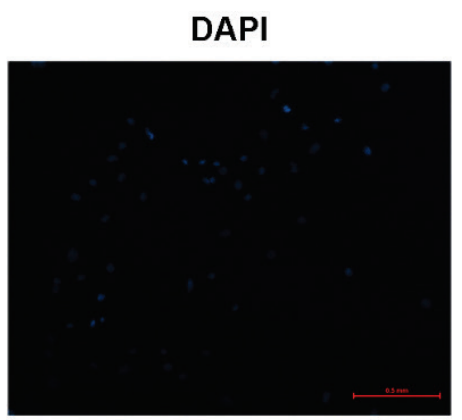

DAPI

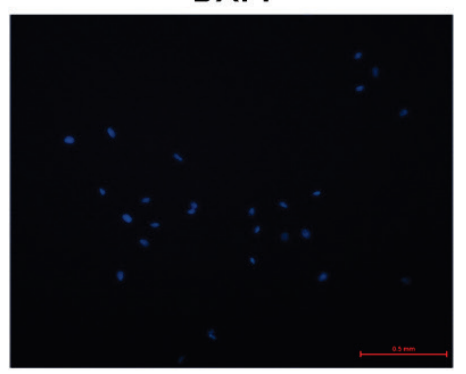

(B)

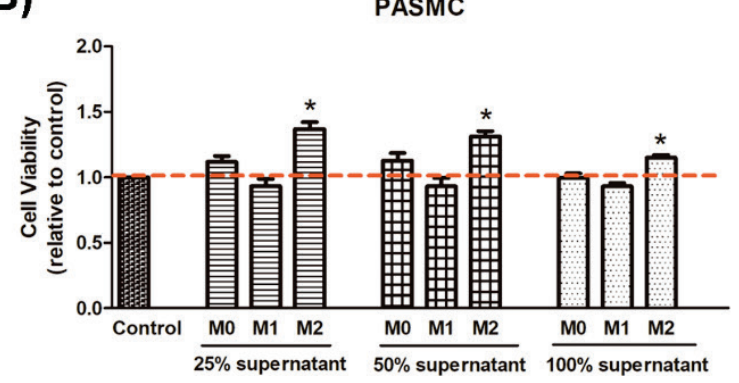

SMA

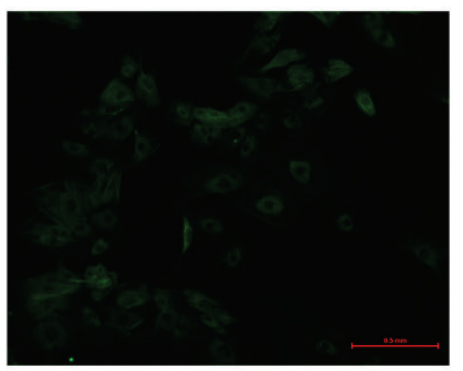

vWF
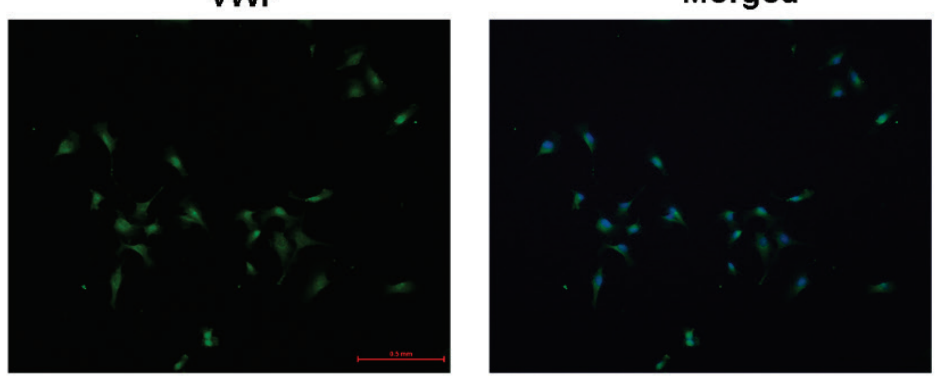

HUVEC

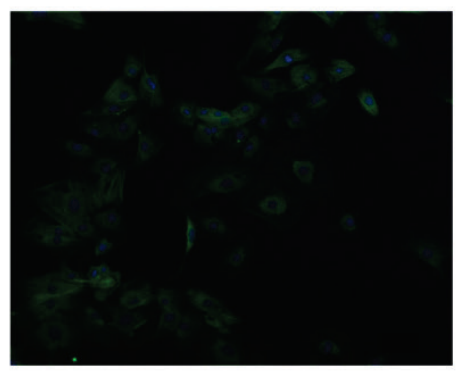

Merged

\section{Merged}

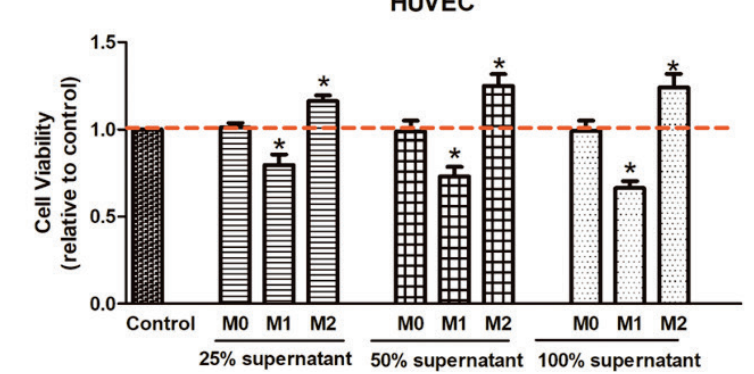

Fig. 5. Cell identification and cell viability determined by Cell Counting Kit-8 (CCK8) assay. (A) Pulmonary arterial smooth muscle cells (PASMCS) and human umbilical vein endothelial cells (HUVECs) were identified by a-smooth muscle actin (SMA) and von Willebrand factor (VWF), respectively. Immunofluorescence images showed there was nearly $100 \%$ purity of these cells. Scale bars $=500 \mu \mathrm{m}$. (B) Evaluation of PASMCs and HUVECs cell viability determined by CCK8 after culturing with different macrophages conditioned medium for 3 days. Supernatants were diluted 1:0, 1:1 and 1:3 with normal culture medium to generate $100 \%, 50 \%$ and $25 \%$ supernatants and daily refreshed. Cells stimulated with normal culture medium were set as control. Data represent means \pm standard deviation. $* \mathrm{P}<0.05$ vs. Control group (tested by independent $\mathrm{t}$ tests).

To further explore the reason for the increase/decrease in cell viability, we examined the effect of activated macrophages on cell cycle and cell apoptosis of PASMC as well as HUVEC. As shown in Fig. 6A, an indirect co-culture model using a combination of PASMC (or HUVEC) and different activated macrophages was used. Upon long term co-culturing, cell cycle analysis showed M1 macrophage blocked HUVEC cell cycle in the G0/G1 phase (dramatic accumulation) while had no effect on PASMC cell cycle. After co-cultured with M2 macrophage, either PASMC or HUVEC had a significant increased percentage of $\mathrm{S}$ and G2/M phases (Fig. 6B/C).

As illustrated in Fig. 6D/E, HUVEC co-cultured 
with M1 macrophage showed a significant increasing apoptosis rate when compared with normal control and M0-treated macrophage. Meanwhile, HUVEC co-cultured with M2 macrophage showed a significant decreased apoptosis rate. That is to say, M1 macrophages promote ECs apoptosis while M2 macrophages had the opposite effect. Nevertheless, under the stimulation of whatever kind of activated macrophages (M1 and M2 macrophage), the apoptosis rate of PASMC showed no difference. These different biologic effects of macrophages might partially explain the temporal difference of polarized macrophages in the pathogenesis of vascular remodelling.

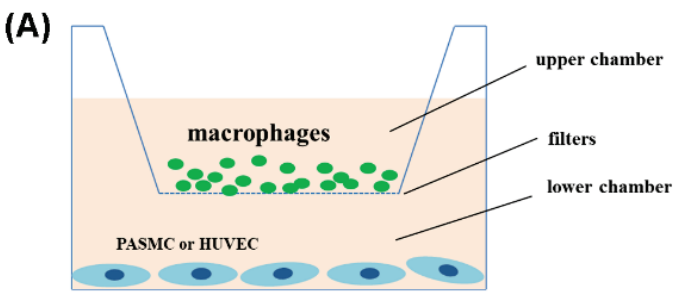

(B)
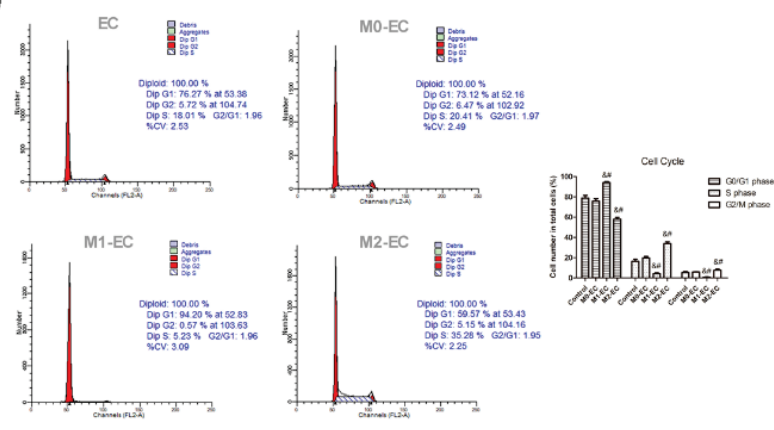

(D)
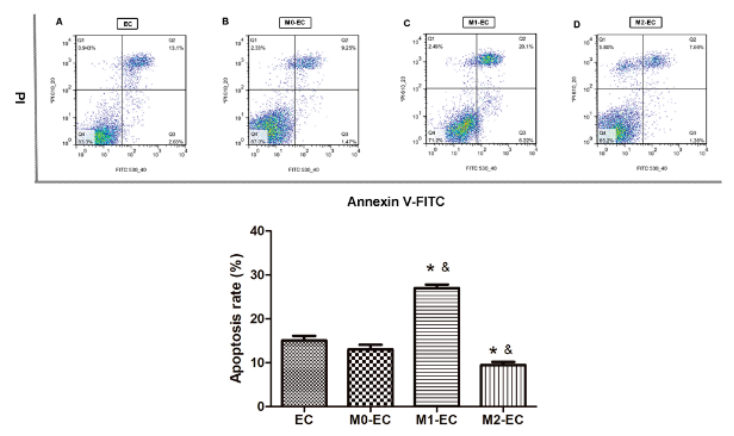

(C)

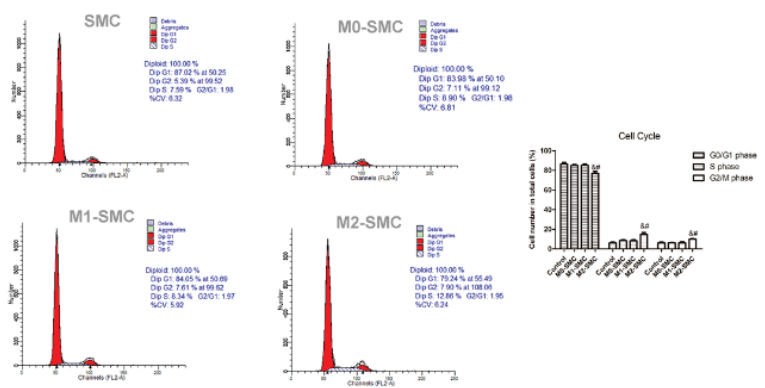

(E)
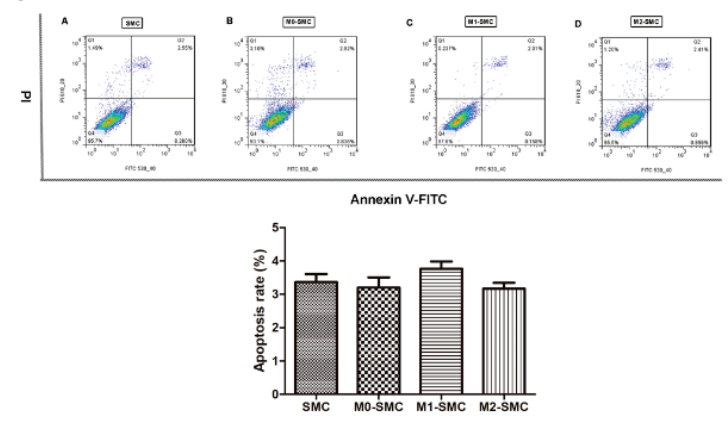

Fig. 6. Effect of different activated macrophages on cell apoptosis and cell cycle of PASMCs and HUVECs. (A) The transwell co-culture system was established in 6-well plate. Macrophages were inserted in the upper chamber while HUVEC or PASMC in the lower chamber. (B) Representative figures showing effect of different activated macrophages on HUVEC cell cycle. M1 macrophage blocked HUVEC cell cycle at G0/G1 phase while M2 macrophage promoted HUVEC cell cycle (dramatic accumulation of $S$ and G2/M phases). (C) Representative figures showing effect of different activated macrophages on PASMC cell cycle. M1 macrophage had no effect on PASMC cell cycle while M2 macrophage promoted PASMC cell cycle (dramatic accumulation of S and G2/M phases), \& $<0.05$ vs. normal control cell (tested by independent $\mathrm{t}$ tests). ${ }^{\#} \mathrm{P}<0.05 \mathrm{vs}$. M0 macrophage (tested by independent $\mathrm{t}$ tests). (D) Representative figures showing effect of different activated macrophages on HUVEC cell apoptosis. HUVECs co-cultured with M1 macrophage showed a significant increasing apoptosis rate while co-cultured with M2 macrophage showed a significant decreasing apoptosis rate. (E) Representative figures showing effect of different activated macrophages on PASMC cell apoptosis. Both M1 and M2 macrophages had little influence on the apoptosis rate of PASMC. Data represent mean \pm standard deviation. ${ }^{*} \mathrm{P}<0.05$ vs. normal control cell (tested by independent $t$ tests). ${ }^{\text {\& }} \mathrm{P}<0.05$ vs. M0 macrophage (tested by independent $t$ tests).

\section{Discussion}

$\mathrm{PAH}$ is a devastating cardiopulmonary disease characterized by vascular remodeling in pulmonary arteries, leading to eventual right heart failure. Currently, advances of therapies significantly improve clinical outcomes of PAH patients, but have little impact on the progression of pulmonary vascular remodeling 
(Thenappan et al. 2018, Davenport et al. 2018). Inflammation was acknowledged as an area of emerging interest with a notation about 'the critical contribution of the immune system in terms of immune surveillance of the vascular milieu' (Erzurum et al. 2010). Consequently, an increasing number of studies have reported the essential roles of inflammation and altered immunity in PAH (Rabinovitch et al. 2014). Macrophages have been widely investigated and represented the most abundant perivascular and lung infiltrating inflammatory cells (Savai et al. 2012). Although a variety of preclinical animal models were used to study PAH (Group $1 \mathrm{PH}$ ) pathogenesis, no model mimics exactly all the features of PAH. Among which, monocrotaline (MCT) animal model is most commonly used by researchers to investigate $\mathrm{PAH}$ pathophysiology due to offering some advantages of mimic several key aspects of human $\mathrm{PAH}$, including vascular remodeling, proliferation of smooth muscle cells, endothelial dysfunction, upregulation of inflammatory cytokines, and right ventricle failure (Maarman et al. 2013, Nogueira-Ferreira et al. 2015). It was reported that, in several established PAH animal models, M2 macrophage has been implicated in the pathogenesis of PAH. Immunization with certain antigens evokes pulmonary arterial muscularization along with a Th2 response and the subsequent polarization of M2 macrophages (Daley et al. 2008). At the meanwhile, a study in mice with hypoxia-induced pulmonary hypertension (Group $3 \mathrm{PH}$ ) suggested that early recruitment of macrophages were critical for the development of $\mathrm{PH}$ and macrophages acquired an alternatively activated phenotype (M2) in response to hypoxia (Vergadi et al. 2011), suggesting a role of microenvironment in deciding macrophage phenotype.

In the present study, the results revealed that CD68 positive macrophages in $\mathrm{PAH}$ lung tissue were significantly elevated and mainly observed in perivascular space and alveolar space. These cells accumulated gradually over time. Following that, it was found for the first time that the pro-inflammatory CD68+NOS2+ M1-like macrophages appeared predominantly in the early stages after MCT injection and were almost absent after 2 weeks. Meanwhile, antiinflammatory CD68+CD206+ M2-like macrophages recruited and accumulated over time in the lung tissue from early to late stages (1st-4th week). Besides, we also analyzed protein level of macrophage-polarized signatures by Western blot and IHC. The findings were generally consistent with the preceding immunofluorescence results, which was M1 macrophage elevated in the early stage while M2 macrophage accumulated mainly in the mid-late stage. This is a very interesting phenomenon and the underlying interpretation is poorly elucidated till now. In fact, in our further functional study, we preliminarily found that M1 macrophages could significantly promote endothelial cell apoptosis. Considering the key role of endothelial injury and followed cell apoptosis in the initiation of pulmonary vascular inflammation (Nogueira-Ferreira, Ferreira, and Henriques-Coelho 2014), we speculate that M1 macrophage might have an important role in the initiation of $\mathrm{PAH}$ pathogenesis. Meanwhile, M2 macrophages were found to promote the proliferation of both SMC and EC, which are key features of vascular remodeling. Previous studies also revealed that M2 macrophage mediated cell proliferation and angiogenesis in cancer cells by releasing a number of growth factors (Tjiu et al. 2009, Yuan et al. 2015).

Conventionally, many diseases are associated with an altered balance of M1/M2 macrophage phenotypes. For example, in atherosclerosis, it is well established that macrophages play important roles in all stages of this disease, from the initiation of lesions and lesion expansion, to the necrosis leading to rupture. A comprehensive review have illustrated that the M1/M2 balance in atherosclerosis plaques to be dynamic, with M1 predominating in disease progression and M2 in regression (Peled and Fisher 2014). Another example is type 2 diabetes in which chronic tissue inflammation is an important pathogenic mediator for disease development. A study from Cucak et al. revealed that proinflammatory M1-like macrophages invade diabetic islets firstly, and then the phenotypes of macrophage changed from an initial proinflammatory (M1 macrophage) toward a profibrotic phenotype (M2 macrophage) over time (Cucak et al. 2014). Similar phenomenon was also found in several other diseases such as cancer (Sica and Bronte 2007), infectious diseases (Davis et al. 2013), multiple sclerosis (Herder et al. 2015), spinal cord injury (Chen et al. 2015) and psychiatric disorders (Nakagawa and Chiba 2014). Blockade of macrophage products, or deactivate macrophage polarization have proved to be fruitful in treating certain diseases. Therefore, studies focus on elucidating specific lung macrophage populations in the pathogenesis of PAH may contribute to provide new insights into the development of new technologies, including macrophage directed therapeutic targets as well 
as diagnostic tools.

Of note, there are several limitations in this study. Firstly, a very interesting phenomenon was noted that mPAP stays low for the first 2 weeks, while suddenly jumps up. Although precise interpretations were still unclear, similar results were reported by Meyrick et al. that a sufficient increase to drive right ventricular reconstruction has not occurred until at least 3 weeks after monocrotaline, and muscularized vessels and medial thickness first becomes significant at 3 weeks (Meyrick, Gamble, and Reid 1980, West and Hemnes 2011). It was largely speculated that a compensatory adjustment may occurred at early stage, and pulmonary arterial pressure will not increase before distinct pulmonary vascular remodelling. Secondly, in vitro experiments in present study is relatively simple and imperfect. A frequentlyused murine macrophage cell line, RAW264.7, was chosen for in vitro functional study, while HUVECs are vein endothelial cells originating from other parts of the vasculature. Nevertheless, our data preliminarily give some implications and explanations for different polarized macrophage in how they may participate in the pathogenesis of PAH vascular remodelling. Basically, more pathophysiologic studies in this field are needed.

\section{Conclusions}

To the best of our knowledge, we present for the first time a temporal dynamic change of pulmonary macrophages polarization status during the development of experimental PAH rat model. M1 macrophages participated in the initial stage of inflammation by accelerating apoptosis of endothelial cell, while M2 macrophages predominated in the reparative stage of inflammation and the followed aberrant tissue remodeling stage by promoting proliferation of both smooth muscle cell and endothelial cell. It is noteworthy that the contributions of M1 and M2 macrophage for pulmonary vascular remodeling in vivo remain largely unclear, and our ex vitro study can only partially generate little implications in this field. Future studies need to be designed to examine this hypothesis perhaps by selectively depleting different activated macrophages at different stages during the development of PAH. Whether macrophages also act as a key player in other types of $\mathrm{PH}$ was indeed to be further investigated.

\section{Conflict of Interest}

There is no conflict of interest.

\section{Acknowledgements}

We want to thank Doctor Yongrui Jia from Center of Medical and Health Analysis faculty of Peking University Health Science Center for her technical assistance.

ZL Zhang designed the study. Y Fan, D Gao and L Gao performed the experiments. YJ Hao and GT Li conducted data analysis. Y Fan and YJ Hao prepared the manuscript. All authors read the manuscript and approved the version to be published.

This work was supported by Scientific Research Seed Fund of Peking University First Hospital (Grant No. 2020SF02) and National Natural Science Foundation of China (Grant No. 82000060 and 91442120).

\section{References}

CHEN YJ, ZHU H, ZHANG N, SHEN L, WANG R, ZHOU JS, HU JG, LU HZ: Temporal kinetics of macrophage polarization in the injured rat spinal cord, J Neurosci Res 93: 1526-33, 2015. https://doi.org/10.1002/jnr.23612

CUCAK H, GRUNNET LG, ROSENDAHL A: Accumulation of M1-like macrophages in type 2 diabetic islets is followed by a systemic shift in macrophage polarization, J Leukocyte Biol 95: 149-160, 2014. https://doi.org/10.1189/jlb.0213075

DALEY E, EMSON C, GUIGNABERT C, DE WAAL MALEFYT R, LOUTEN J, KURUP V P, HOGABOAM C, TARASEVICIENE-STEWART L, VOELKEL N F, RABINOVITCH M, GRUNIG E, GRUNIG G: Pulmonary arterial remodeling induced by a Th2 immune response. J Exp Med 205: 361-372, 2008. https://doi.org/10.1084/jem.20071008

DAVENPORT A P, KUC R E, SOUTHAN C, MAGUIRE JJ: New drugs and emerging therapeutic targets in the endothelin signaling pathway and prospects for personalized precision medicine. Physiol Res 67: S37-S54, 2018. https://doi.org/10.33549/physiolres. 933872 
DAVIS MJ, TSANG TM, QIU Y, DAYRIT JK, FREIJ J B, HUFFNAGLE GB, OLSZEWSKI MA: Macrophage M1/M2 polarization dynamically adapts to changes in cytokine microenvironments in Cryptococcus neoformans infection. MBio 4: e00264-13, 2013. https://doi.org/10.1128/mBio.00264-13

ERZURUM S, ROUNDS S I, STEVENS T, ALDRED M, ALIOTTA J, ARCHER S L, ASOSINGH K, BALABAN R, BAUER N, BHATTACHARYA J, BOGAARD H, CHOUDHARY G, DORN GW, DWEIK R, FAGAN K, FALLON M, FINKEL T, GERACI M, GLADWIN M T, HASSOUN P M, HUMBERT M, KAMINSKI N, KAWUT S M, LOSCALZO J, MCDONALD D, MCMURTRY IF, NEWMAN J, NICOLLS M, RABINOVITCH M, SHIZURU J, OKA M, POLGAR P, RODMAN D, SCHUMACKER P, STENMARK K, TUDER R, VOELKEL N, SULLIVAN E, WEINSHILBOUM R, YODER MC, ZHAO Y, GAIL D, MOORE TM: Strategic plan for lung vascular research: An NHLBI-ORDR Workshop Report. Am J Respir Crit Care Med 182: 1554-1562, 2010. https://doi.org/10.1164/rccm.201006-0869WS

FARKAS D, THOMPSON AAR, BHAGWANI AR, HULTMAN S, JI H, KOTHA N, FARR G, ARNOLD ND, BRAITHWAITE A, CASBOLT H, COLE JE, SABROE I, MONACO C, COOL CD, GONCHAROVA EA, LAWRIE A, FARKAS L: Toll-like receptor 3 is a therapeutic target for pulmonary hypertension. Am J Respir Crit Care Med 199: 199-210, 2019. https://doi.org/10.1164/rccm.201707-13700C

FREIRE-DE-LIMA CG, XIAO YQ, GARDAI SJ, BRATTON DL, SCHIEMANN W P, HENSON PM: Apoptotic cells, through transforming growth factor-beta, coordinately induce anti-inflammatory and suppress pro-inflammatory eicosanoid and NO synthesis in murine macrophages. J Biol Chem 281: 38376-38384, 2006. https://doi.org/10.1074/jbc.M605146200

FRID MG, BRUNETTI JA, BURKE DL, CARPENTER TC, DAVIE NJ, REEVES JT, ROEDERSHEIMER MT, VAN ROOIJEN N, STENMARK KR: Hypoxia-induced pulmonary vascular remodeling requires recruitment of circulating mesenchymal precursors of a monocyte/macrophage lineage. Am J Pathol 168: 659-669, 2006. https://doi.org/10.2353/ajpath.2006.050599

GAO L, FAN Y, HAO Y, YUAN P, LIU D, JING Z, ZHANG Z: Cysteine-rich 61 (Cyr61) upregulated in pulmonary arterial hypertension promotes the proliferation of pulmonary artery smooth muscle cells. Int J Med Sci 14: 820-828, 2017. https://doi.org/10.7150/ijms.19282

HERDER V, ISKANDAR C D, KEGLER K, HANSMANN F, ELMARABET S A, KHAN M A, KALKUHL A, DESCHL U, BAUMGARTNER W, ULRICH R, BEINEKE A: Dynamic changes of microglia/macrophage M1 and M2 polarization in theiler's murine encephalomyelitis, Brain Pathol, 25: 712-723, 2015. https://doi.org/10.1111/bpa.12238

MAARMAN G, LECOUR S, BUTROUS G, THIENEMANN F, SLIWA K: A comprehensive review: the evolution of animal models in pulmonary hypertension research; are we there yet? Pulm Circ 3: 739-756, 2013. https://doi.org/10.1086/674770

MEYRICK B, GAMBLE W, REID L: Development of Crotalaria pulmonary hypertension: hemodynamic and structural study. Am J Physiol 239: H692-H702, 1980. https://doi.org/10.1152/ajpheart.1980.239.5.H692

MURRAY PJ, ALLEN JE, BISWAS SK, FISHER EA, GILROY DW, GOERDT S, GORDON S, HAMILTON JA, IVASHKIV LB, LAWRENCE T, LOCATI M, MANTOVANI A, MARTINEZ FO, MEGE JL, MOSSER DM, NATOLI G, SAEIJ JP, SCHULTZE JL, SHIREY KA, SICA A, SUTTLES J, UDALOVA I, VAN GINDERACHTER JA, VOGEL SN, WYNN TA: Macrophage activation and polarization: nomenclature and experimental guidelines. Immunity 41: 14-20, 2014. https://doi.org/10.1016/j.immuni.2014.06.008

NAKAGAWA Y, CHIBA K: Role of microglial M1/M2 polarization in relapse and remission of psychiatric disorders and diseases. Pharmaceuticals (Basel) 7: 1028-1048, 2014. https://doi.org/10.3390/ph7121028

NOGUEIRA-FERREIRA R, FERREIRA R, HENRIQUES-COELHO T: Cellular interplay in pulmonary arterial hypertension: implications for new therapies. Biochim Biophys Acta 1843: 885-893, 2014. https://doi.org/10.1016/j.bbamcr.2014.01.030

NOGUEIRA-FERREIRA R, VITORINO R, FERREIRA R, HENRIQUES-COELHO T: Exploring the monocrotaline animal model for the study of pulmonary arterial hypertension: A network approach. Pulm Pharmacol Ther 35: 8-16, 2015. https://doi.org/10.1016/j.pupt.2015.09.007

PELED M, FISHER EA: Dynamic aspects of macrophage polarization during atherosclerosis progression and regression. Front Immunol 5: 579, 2014. https://doi.org/10.3389/fimmu.2014.00579 
RABINOVITCH M, GUIGNABERT C, HUMBERT M, NICOLLS MR: Inflammation and immunity in the pathogenesis of pulmonary arterial hypertension, Circ Res 115: 165-175, 2014. https://doi.org/10.1161/CIRCRESAHA.113.301141

SAKAO S, TARASEVICIENE-STEWART L, WOOD K, COOL CD, VOELKEL NF: Apoptosis of pulmonary microvascular endothelial cells stimulates vascular smooth muscle cell growth. Am J Physiol Lung Cell Mol Physiol 291: L362-L368, 2006. https://doi.org/10.1152/ajplung.00111.2005

SAVAI R, PULLAMSETTI SS, KOLBE J, BIENIEK E, VOSWINCKEL R, FINK L, SCHEED A, RITTER C, DAHAL BK, VATER A, KLUSSMANN S, GHOFRANI HA, WEISSMANN N, KLEPETKO W, BANAT GA, SEEGER W, GRIMMINGER F, SCHERMULY RT: Immune and inflammatory cell involvement in the pathology of idiopathic pulmonary arterial hypertension. Am J Respir Crit Care Med 186: 897-908, 2012. https://doi.org/10.1164/rccm.201202-03350C

SICA A, BRONTE V: Altered macrophage differentiation and immune dysfunction in tumor development. J Clin Invest 117: 1155-1166, 2007. https://doi.org/10.1172/JCI31422

TABAS I, BORNFELDT KE: Macrophage Phenotype and Function in Different Stages of Atherosclerosis. Circ Res 118: 653-667, 2016. https://doi.org/10.1161/CIRCRESAHA.115.306256

THENAPPAN T, GOEL A, MARSBOOM G, FANG Y H, TOTH P T, ZHANG H J, KAJIMOTO H, HONG Z, PAUL J, WIETHOLT C, POGORILER J, PIAO L, REHMAN J, ARCHER SL: A central role for CD68(+) macrophages in hepatopulmonary syndrome. Reversal by macrophage depletion. Am J Respir Crit Care Med 183: 1080-1091, 2011. https://doi.org/10.1164/rccm.201008-1303OC

THENAPPAN T, ORMISTON ML, RYAN JJ, ARCHER SL: Pulmonary arterial hypertension: pathogenesis and clinical management. BMJ 360: j5492, 2018. https://doi.org/10.1136/bmj.j5492

TIAN W, JIANG X, TAMOSIUNIENE R, SUNG YK, QIAN J, DHILLON G, GERA L, FARKAS L, RABINOVITCH M, ZAMANIAN RT, INAYATHULLAH M, FRIDLIB M, RAJADAS J, PETERS-GOLDEN M, VOELKEL NF, NICOLLS MR: Blocking macrophage leukotriene b4 prevents endothelial injury and reverses pulmonary hypertension. Sci Transl Med 5: 200ra117, 2013. https://doi.org/10.1126/scitranslmed.3006674

TJIU JW, CHEN JS, SHUN CT, LIN SJ, LIAO YH, CHU CY, TSAI TF, CHIU HC, DAI YS, INOUE H, YANG PC, KUO ML, JEE SH: Tumor-associated macrophage-induced invasion and angiogenesis of human basal cell carcinoma cells by cyclooxygenase-2 induction. J Invest Dermatol 129: 1016-1025, 2009. https://doi.org/10.1038/jid.2008.310

VERGADI E, CHANG MS, LEE C, LIANG OD, LIU X, FERNANDEZ-GONZALEZ A, MITSIALIS SA, KOUREMBANAS S: Early macrophage recruitment and alternative activation are critical for the later development of hypoxia-induced pulmonary hypertension. Circulation 123: 1986-1995, 2011. https://doi.org/10.1161/CIRCULATIONAHA.110.978627

WEST J, HEMNES A: Experimental and transgenic models of pulmonary hypertension. Compr Physiol 1: 769-782, 2011. https://doi.org/10.1002/cphy.c100003

YUAN A, HSIAO YJ, CHEN HY, CHEN HW, HO CC, CHEN YY, LIU YC, HONG TH, YU SL, CHEN JJ, YANG P C: Opposite effects of M1 and M2 macrophage subtypes on lung cancer progression. Sci Rep 5: 14273, 2015. https://doi.org/10.1038/srep14273

ZALOUDIKOVA M, VYTASEK R, VAJNEROVA O, HNILICKOVA O, VIZEK M, HAMPL V, HERGET J: Depletion of alveolar macrophages attenuates hypoxic pulmonary hypertension but not hypoxia-induced increase in serum concentration of MCP-1. Physiol Res 65: 763-768, 2016. https://doi.org/10.33549/physiolres.933187 